\section{INCREASED INSTITUTIONAL SURGICAL EXPERIENCE IN ROBOT-ASSISTED RADICAL HYSTERECTOMY FOR EARLY STAGE CERVICAL CANCER REDUCES RECURRENCE RATE. RESULTS FROM A NATIONWIDE STUDY}

${ }^{1}$ Linnea Ekdahl, ${ }^{2}$ Emelie Wallin, ${ }^{3}$ Emilia Alfonzo, ${ }^{1}$ Petur Reynisson, ${ }^{1}$ Celine Lönnerfors, ${ }^{3}$ Pernilla Dahm-Kähler, ${ }^{2}$ Henrik Falconer, 'Jan Persson. ' Skåne University Hospital and Lund University Faculty of Medicine; ${ }^{2}$ Karolinska Institutet; ${ }^{3}$ Sahlgrenska Academy at University of Gothenburg

\subsection{6/ijgc-2020-ESG0.206}

Introduction/Background The aim of this study was to evaluate the impact of institutional surgical experience on recurrence following robotic radical hysterectomy (RRH) for early stage cervical cancer.

Methodology All women in Sweden who underwent an RRH for stage IA2-IB1 cervical cancer at tertiary referral centers from its implementation in December 2005 until June 2017 were identified using a Swedish nationwide register and local hospital registers. Registry data was controlled by a chart review on all women. Recurrence rates and pattern of recurrence was compared between early and late ( $\leq 50$ vs $>50$ procedures) institutional series.

Results 635 women were included. Regression analysis identified a lower risk of recurrence with increased experience but without a clear cut off level. Among the 489 women who did not receive adjuvant radio chemotherapy (RC-T), the rate of recurrence was $3.6 \%$ in the experienced cohort $(>50$ procedures) compared to $9.3 \%$ in the introductory cohort $(\mathrm{p}<0.05)$. This was also seen in tumors $<2 \mathrm{~cm}$ regardless of RC-T $(p<0.05)$ whereas no difference in recurrence was seen when analyzing all women receiving RC-T.

Conclusion In conclusion, the rate of recurrence following RRH for early stage cervical cancer decreased with increased institutional surgical experience, in tumors $<2 \mathrm{~cm}$ and in women who did not receive adjuvant RC-T.

Disclosures Jan Persson, Henrik Falconer and Celine Lönnerfors have received honoraria for lectures and proctoring in robotic surgery, all outside the presented research. The other authors have no conflicts of interest.

\section{LONG-TERM RESULTS OF NEOADJUVANT DOSE-DENSE PLATINUM-BASED CHEMOTHERAPY IN PATIENTS WITH LOCALLY ADVANCED CERVICAL CANCER}

${ }^{1}$ Olga Smirnova, ${ }^{2}$ Elena Ulrikh, ${ }^{1}$ Alina Abramova, ${ }^{1}$ Maria Yakovleva, ${ }^{1}$ Anna Petrova, ${ }^{1}$ Nikolay Mikaya, ${ }^{1}$ Olga Lavrinovich, ${ }^{1}$ Nikolay Bondarev, ${ }^{1}$ Adel Urmancheeva, ${ }^{1}$ Igor Berlev. ${ }^{1}$ N.N. Petrov National Medical Research Center of Oncology; ${ }^{2}$ Almazov National Medical Research Centre; North-Western State Medical University, N.N.Petrov National Medical Research Centre of Oncology; Oncology

\subsection{6/ijgc-2020-ESGO.207}

Introduction/Background In our study, we evaluated the longterm results of neoadjuvant dose-dense platinum-based chemotherapy in patients with locally advanced cervical cancer FIGO IB2, IIB stage.

Methodology A cohort of 119 consecutive patients with median age of 43 (range 27-68) years was studied. All patients had verified locally-advanced $\left(\mathrm{cT} 1_{\mathrm{B}} 2 \mathrm{Nx}, 0 \mathrm{M} 0\right.$; $\mathrm{cT} 2{ }_{\mathrm{B}} \mathrm{Nx}, 0 \mathrm{M} 0$ ) cervical cancer and received 3 dose-dense intravenous neoadjuvant AP (cisplatin $75 \mathrm{mg} / \mathrm{m} 2$, doxorubicin 35 $\mathrm{mg} / \mathrm{m} 2 ; \mathrm{n}=75$ ) or TP (cisplatin $60 \mathrm{mg} / \mathrm{m} 2$ and paclitaxel 60 $\mathrm{mg} / \mathrm{m} 2 ; \mathrm{n}=30$ ) chemotherapy cycles.
Results The median follow-up was 28 (4 - 48) months for AP and 17 (3 - 30) months for TP group, accordingly. The overall survival rates in FIGO IB2 stage for AP and TP groups were $100 \%$. For FIGO IIB stage the overall survival rate in AP group was 94\%, in TP group - 97\%.

The disease-free survival rate in FIGO IB2 stage for AP group was $78 \%$, for TP group - 100\%. For FIGO IIB stage the disease-free survival rate in the AP group was $87 \%$, in TP group - 100\%.

Conclusion The dose-intensive chemotherapy is an effective treatment modality for locally-advanced cervical cancer and may be a feasible alternative for standard treatment approach. It deserves further study in larger patient cohort with evaluation of the long-term results.

\section{COMPARISON OF TOPICAL TREATMENT OF CERVICAL SQUAMOUS INTRAEPITHELIAL LESIONSWITH IMIQUIMOD WITH STANDARD EXCISIONAL TECHNIQUE USING LLETZ: A RANDOMIZED CONTROLLED TRIAL}

${ }^{1}$ Andrej Cokan, ${ }^{1}$ Maja Pakiž, ${ }^{1} J u r e ~ K n e z, ~{ }^{1}$ Andraž Dovnik, ${ }^{1}$ Tatjana Kodrič, ${ }^{1}$ Leyla Al Mahdawi, ${ }^{2}$ Tamara Serdinšek, ${ }^{3}$ Alenka Repše Fokter, ${ }^{1}$ Igor But. ${ }^{1}$ Umc Maribor Department for Gynaecologic Oncology and Oncology of the Breast; ${ }^{2}$ Umc Maribor: Department for General Gynaecology and Gynaecologic Urology; ${ }^{3}$ Medical Faculty Maribor; Gh Celje

\subsection{6/ijgc-2020-ESG0.208}

Introduction/Background Standard treatment of cervical squamous intraepithelial lesions (SIL) is large loop excision of transformation zone (LLETZ), which is associated with increased risk of preterm delivery, higher subfertility rate, and higher spontaneous abortions rate. Our aim was to determine whether topical treatment of high-grade SIL (HSIL) with imiquimod is comparable to standard treatment in terms of efficiency and side effects occurrence.

Abstract 608 Table 1 Succces of treated in patient treated with imiquimod (group 1) and LLETZ (group 2)

\begin{tabular}{|c|c|c|c|}
\hline & $\begin{array}{l}\text { Group 1 } \\
\text { (Imiquimod) }\end{array}$ & $\begin{array}{l}\text { Group 2 } \\
\text { (LLETZ) }\end{array}$ & p-value \\
\hline CIN 2 [N/total N (\%)] & $17 / 21(81.0)$ & $12 / 19(63.2)$ & 0.366 \\
\hline CIN 3 [N/total N (\%)] & $10 / 22(45.5)$ & $27 / 33(81.8)$ & $0.012^{*}$ \\
\hline All patients [N/total $\mathrm{N}(\%)]$ & $27 / 43(62.8)$ & $39 / 52(75.0)$ & 0.288 \\
\hline
\end{tabular}

Abstract 608 Table 2 Presence of side effects and the highest grade of side effects in patients treated with imiquimod (group 1) and LLETZ (group 2)

\begin{tabular}{|c|c|c|c|c|}
\hline & & $\begin{array}{l}\text { Group } 1 \\
\text { (Imiquimod) }\end{array}$ & $\begin{array}{l}\text { Group } 2 \\
\text { (LLETZ) }\end{array}$ & p-value \\
\hline \multicolumn{2}{|c|}{$\begin{array}{l}\text { Side effects: yes } \\
{[\mathrm{N} / \text { total } \mathrm{N}(\%)]}\end{array}$} & $\begin{array}{l}46 / 52 \\
(88.5 \%) \\
\end{array}$ & $\begin{array}{l}23 / 52 \\
(44.2) \\
\end{array}$ & $<0.001^{*}$ \\
\hline \multirow{4}{*}{$\begin{array}{l}\text { Highest } \\
\text { grade of } \\
\text { side effect } \\
\text { [N/total } N \\
(\%)]\end{array}$} & Grade 1 & $18 / 52(34.6)$ & $\begin{array}{l}14 / 52 \\
(26.9) \\
\end{array}$ & \multirow[t]{4}{*}{$<0.001^{*}$} \\
\hline & Grade 2 & $20 / 52(38.5)$ & $7 / 25(13.5)$ & \\
\hline & Grade 3 & $7 / 52(13.5)$ & $0 / 52(0)$ & \\
\hline & Other & $1 / 52(1.9)$ & $2 / 52(3.8)$ & \\
\hline
\end{tabular}

*Statistically significant difference. 
Methodology We performed a randomized controlled study. Patients with HSIL aged 18-40 years were included and treated with either imiquimod, 3 times per week for 16 weeks (experimental arm), or with LLETZ (control arm). Treatment success was evaluated by regression to low-grade SIL (LSIL) 20 weeks after initiation of the treatment in the experimental arm and by negative cytology 6 months after LLETZ in the control arm. Secondary outcome was occurrence of the side effects during and after treatment. Statistical analysis was performed using SPSS Statistics Programme. Statistical significance was set at $\mathrm{p}$-value $<0.05$.

Results We included 104 patients. In the experimental arm, 43 out of 52 patients $(82.7 \%)$ completed treatment, while in the control arm, all of the 52 patients received the planned treatment (100\%). Treatment with imiquimod was successful in $62.8 \%$ and treatment with LLETZ in $75.0 \%$, the difference was not statistically significant $(p$-value $=0.288)$. When evaluating treatment success in the intermediate risk subgroup (patients with cervical intraepithelial neoplasia grade 2 - CIN 2), there were also no statistically significant differences between groups $(p$-value $=0.366)$. However, LLETZ was significantly more successful in patients with CIN 3 lesions (p-value=0.012). We did not observe any cases of progression of the precancerous disease to cancer. Side effects and severe side effects were significantly more prevalent in the imiquimod than in the LLETZ group $(88.5 \%$ vs. $44.2 \%$ (p-value $<0.001)$ and $51.9 \%$ vs. $13.5 \%$ (pvalue $<0.001$ ), respectively). The most prevalent side effects were vaginal inflammation, flu-like and lower urinary tract symptoms. Over the course of the treatment with imiquimod, overall occurrence and the severity of side effects decreased.

Conclusion Topical imiquimod has a potential of becoming an alternative treatment for HSIL, especially in younger women with intermediate risk HSIL. However, its use is associated with higher occurrence of side effects, which can affect patients' quality of life. In the future, larger studies evaluating the long-term effects of this treatment are needed, especially in the view of disease progression and recurrence.

Disclosures The authors declare no competing interests. This research was financially supported by UMC Maribor.

\section{Diagnostics}

\section{EVALUATION OF MICRO-RNA EXPRESSION IN CYTOLOGICAL SMEARS IN WOMEN WITH LOW GRADE SQUAMOUS INTRAEPITHELIAL LESIONS}

Tatyana Prisyazhnaya, Anastasia Malek, Margarita Knyazeva, Igor Berlev. N.N. Petrov National Medical Research Center of Oncology; North-Western State Medical University Named after I.I. Mechnikov

\subsection{6/ijgc-2020-ESG0.209}

Introduction/Background MicroRNAS are short molecules that regulate gene expression. The microRNA expression profile changes in cells during neoplastic transformation. In particular, characteristic changes in microRNA are observed in the cells of the cervical epithelium during the development of intraepithelial neoplasia. These changes are compounded in invasive cervical cancer cells. Accordingly, microRNAS can serve as diagnostic or prognostic biomarkers in patients with cervical dysplasia of varying severity.

In this study, we analyzed microRNA in patients with low grade squamous intraepithelial lesions (LSIL) and compared the data obtained with the clinical course of the disease.
Methodology Total RNA was isolated from the epithelium of patients with low grade squamous intraepithelial lesions and divided into two pools: "persistence" $(n=10)$ and "recovery" $(n=10)$, depending on the data of repeated cytological examination conducted after 6-9 months. In the obtained samples, we performed a comprehensive screening analysis of 85 micro-RNA expression (Cancer focus miRCURY RT-PCR panel, Exiqon, Denmark).

Results The results of microRNA profiling showed different levels of expression of 9 molecules in the compared groups. In cases of persistent cervical epithelial atypia during dynamic observation, miR-126-3p Mir-16-5p miR-182-5p miR-200c-3p, miR-205-5p, miR-223-3p, miR-24-3p molecules were expressed significantly more actively than in the group of samples obtained from patients whose cervical epithelium condition normalized during observation. The reverse situation was observed for miR-192-5p and miR let-7f-5p.

Conclusion MicroRNA molecules whose expression level correlates with the prognosis of cervical epithelial dysplasia can serve as useful biomarkers and be used to personalize the treatment of this common gynecological disease. Validation of the microRNA estimation method requires more extensive research.

Disclosures The authors declare no conflict of interest. The funders had no role in the design of the study; in the collection, analyses, or interpretation of data; in the writing of the manuscript, or in the decision to publish the results.

\section{DETECTING CERVICAL DYSPLASIA WITH FOLATE RECEPTOR-MEDIATED DETECTION STAINING AGENT}

'Luka Roškar, 'Nataša Borko Tavželj, '2David Moos. 'Murska Sobota General Hospital; Gynecology and Obstetrics Department; ${ }^{2}$ Uag School of Medicine

\subsection{6/jgc-2020-ESG0.210}

Introduction/Background Colposcopy is an essential method in the diagnosis of precancerous lesions. In recent years, new adjunctive technologies have been emerging with an aim to increase the effectiveness of colposcopy. In our randomized pilot study, we tested the Folate Receptor-mediated Detection staining solution (FRD) on 10 patients who visited our colposcopy outpatient office.

Abstract 619 Table 1 Results of FRD staining, PAP smear, HPV test, colposcopy and histology in 10 patients.

\begin{tabular}{llllll}
\hline Case & FRD & PAP & HPV & $\begin{array}{l}\text { Colposcopy } \\
\text { (Swede score) }\end{array}$ & Histology \\
\hline 1 & + & H-SIL & + & 5 & CIN 1 \\
2 & + & H-SIL & + & 8 & CIN 2 \\
3 & + & H-SIL & - & 4 & No dysplasia \\
4 & + & ASC-H & + & 6 & CIN 2 \\
5 & + & H-SIL (2x) & + & 8 & Acute cervicitis \\
6 & - & H-SIL & - & 0 & Chronic Cervicitis \\
7 & + & PAP B (2x) & + & 4 & CIN 1 \\
8 & + & PAP A & + & 3 & CIN 1 \\
9 & + & L-SIL (2x) & + & 3 & CIN 1 \\
10 & - & ASC-US & - & 3 & No dysplasia
\end{tabular}

